$\xi=-1$ 国

\title{
Hybrid RF/visible light communication in downlink wireless system
}

\author{
Tharindu D. Ponnimbaduge Perera ${ }^{1}$, Akashkumar Rajaram ${ }^{1}$, Sangay Chedup ${ }^{2}$, \\ Dushantha Nalin K. Jayakody ${ }^{1 *}$, Bin Chen ${ }^{3}$
}

\author{
${ }^{I}$ School of Computer Science and Robotics, National Research Tomsk Polytechnic University, RUSSIA \\ ${ }^{2}$ Department of Electronics and Communication Engineering, Jigme Namgyel Engineering College, BHUTAN \\ ${ }^{3}$ School of Computer and Information, Hefei University of Technology (HFUT), Hefei, CHINA \\ *Corresponding author E-mail:nalin@tpu.ru
}

\begin{abstract}
The spectrum crunch in Radio Frequency (RF) Communication and the prospect of Visible Light Communication (VLC) as an alternative to RF has made way for high speed VLC deployment. The ubiquitous coverage of RF and the high speed data rate achieved with VLC, make the two technologies complimentary to each other, making the hybrid RF/VLC an emerging trend in wireless networks. This study presents a downlink communication of a hybrid RF-VLC, where the signal received through RF channel by a relay is decoded and the symbols are converted into an electrical signals. The electrical signal is fed to an array of LEDs in the relay where data is transmitted in rays of light from LEDs. The hybrid RFVLC system performance is compared with a conventional RF system to understand the performance difference between the two communication models. Thus, the performance of both system models are analysed in terms of Symbol Error Rate and Outage Probability. Numerical results show that the proposed hybrid RF-VLC system is superior to conventional lone RF cooperative system.
\end{abstract}

Keywords: Hybrid RF-VLC, Outage Probability, Radio Frequency, Visible Light, $5 G$

\section{Introduction}

The exponential growth of demand for global internet data access is severely questioning the sustainability of Radio Frequency (RF) spectrum. The global internet demand has reached 2.5 exabytes per month in 2014 [1] and exponential deployment of internet of things (IoT) devices in the network is leading to RF spectrum exhaustion as predicted by 2020 [2] [3]. The wider coverage of RF wireless communication has few more problems like its security vulnerability for deployment in heightened security and its hazardous nature in applications such as business, industries, hospitals, and armed forces. To counter the limitations of RF, visible light communication (VLC), a technology which enables the usage of energy efficient lighting luminaries, particularly the light emitting diodes (LEDs) as high speed data access points (APs) offers an alternative to RF [1], [2], [4], [5]. The deployment of VLC is an ideal choice for indoor environment like high security smart rooms where high speed data is transmitted by modulating [5] the varying light intensity imperceptible to human eyes. The unregulated license free visible light (VL) spectrum with provision of hundreds of terahertz bandwidth [1], enhanced by its application prospect on existing infrastructure, makes the VLC a viable choice. On the other hand, standalone VLC has limitation on network coverage while RF provides ubiquitous service [1], [5]. VLC has the potential to reach the RF unreached dead zones in RF restricted applications. These factors have encouraged the merging of RF and VLC technologies exploiting their complementarity as hybrid RF/VLC [6], [7]. The high speed data rate in Gbps, license free spectrum, enhanced security and almost zero interference is achieved by VLC [4]. RF technology enhances coverage conforming to the benefit of hybrid RF/VLC as envisioned to achieve the objective of future high speed data fifth generation $(5 \mathrm{G})$ networks. The prospect of its deployment in existing infrastructure also promotes an energy efficient, sustainable and green communication technology. In the work of [8], the authors have performed outage probability analysis of hybrid VLC scheme in comparison with ordinary QAM scheme. Considering the prospect of VLC and hybrid VLC/RF, these fields have resulted in a paradigm shift of research interest in wireless communication particularly towards promoting green communication [9]. Hybrid VLC/RF communication systems in [1] examines for indoor environments to provide enhanced coverage where VLC plays a major role and RF system fill-up the coverage holes. Authors of [10], [11] introduce dualhop VLC/RF system as a new approach to extend the coverage of VLC with optimal DC bias for transmitted subcarrier intensity modulation (SIM) signal to transmit information over a VLC channel to a relay. The relay is enabled with energy harvesting (EH) to transmit received data through RF channel. The QoS in wireless network is enhanced with RF and VLC through link selection process, transmitting a data over a link which in turn guarantees the desired QoS [12]. In [13], the authors consider a combined light fidelity (Li-Fi) with the conventional RF system where load balancing scheme based on game theory is proposed to navigate the network access in strong blockage scenario. Compare to analysis of existing hybrid VLC/RF schemes [14], [15], the authors of the work [5] investigated the enactment of power efficiency in hybrid VLC/RF scheme using resource allocation algorithms.

In this paper, we concentrate on the design of a downlink hybrid RF-VLC system in wireless communication, consisting of a 
source, a destination, and a Decode-Forward (DF) relay. Source transmits the signal to relay using RF signals, while the relay uses VLC channel to transmit the signal to destination. The data transmitted in RF channel is decoded and converted to an electrical signal, then feeds the LED array, hence RF data is converted to an electrical signal. LEDs relay transmits the signal through VLC channel by modulating the varying intensity (i.e. flickering of light is data transmission in 1's and 0's) of visible light emitted from VLC relay (LEDs array). Hence, LED luminaries perform two basic operations i.e. illumination and data transmission. The photo detector or IoT devices at destination receives the rays of illumination and transforms to an electrical signal. The transformed electrical signal is further decoded by performing the analog to digital conversion (ADC) where original RF signal transmitted in VLC channel is retrieved at the destination. The performance of proposed hybrid RF/VLC scheme is analysed by the simulations of two system models: RF source to relay with decoding factor and relay to destination as lone RF data transmission scheme and hybrid RF source to relay with decoding factor and VLC relay to destination links. Quality of service (QoS) performance analysis of these two system models are performed on the basis of outage probability and symbol error rate (SER) against the SNR values. Therefore, this paper considers integrating the RF wireless communication seamlessly into a VLC network for downlink from RF to VLC network as hybrid RF/VLC high speed data wireless network. The paper is further arranged with hybrid RF/VLC system model in section 2, followed by outage probability computation of the RF-VLC hybrid communication system in section 3 . The numerical results are explained in section 4 and conclusion in section 5. The hybrid RF/VLC arrangement perform better than RF communication alone in smart room enabled with energy efficient lighting deployment.

\section{Hybrid RF-VLC system model}

In this paper, we consider a high speed data hybrid RFVLC downlink wireless network for an energy efficient LED enabled smart room or even a conventional meeting room as shown in Figure1. This system consists of RF source, LED relay and a destination. The RF source serves as the transmitter, the information source and the user's mobile device serves as the final destination receiving high speed data from the relay via VLC channel. Moreover, time division relying scheme has been used with the decode-and forward relaying protocol. In the first time slot (T1), the transmitter transmits the RF information signal to the relay placed on the roof of the building. In the second time slot (T2), relay terminal will decode the received signal and re transmit over the VLC channel to user's mobile device located inside the meeting room. Thereby user is able to access the high speed VLC network.

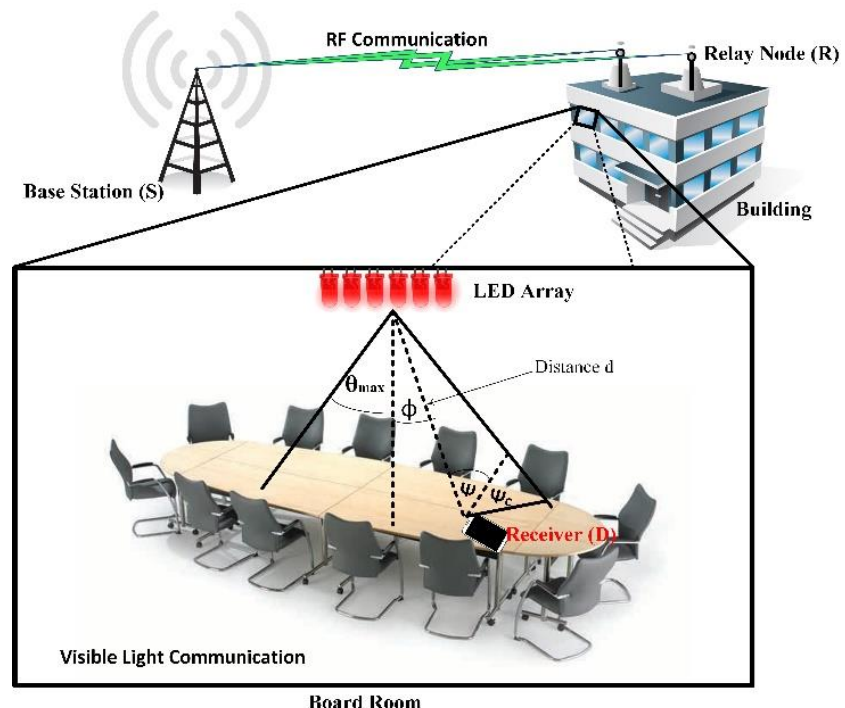

Fig. 1: hybrid RF-VLC transmission system

\subsection{Source to relay RF link}

During T1, the base station (BS) transmits the QPSK modulated signal over Rayleigh flat fading with additive white Gaussian noise (AWGN) channel to the relay. The received signal at the relay from the BS can be written as

$$
y_{s r}=\sqrt{p_{s}} h_{s r} x+n_{s r},
$$

where $p_{s}$ the transmitted power at the base station, $h_{s r}$ is denotes the Rayleigh flat channel fading co-efficient from the BS to relay, $n_{s r}$ represents the AWGN with zero mean and $x$ denotes the QPSK modulated information symbols. Thus the received SNR at the relay after the BS's transmission can be written as

$$
\gamma_{s r}=p_{s}\left|h_{s r}\right|^{2}+N_{o},
$$

where $N_{o}$ is the power of noise suffered by receiver in the relay. Similarly for the equation (1) and (2), received signal at the destination $\left(y_{s r}\right)$ and the SNR of the received signal $\left(\gamma_{s r}\right)$ at the destination can be formulated in a conventional RF cooperative system. The SER and outage results of the conventional RF cooperative system have been used in the Section IV to compare with the proposed RF-VLC hybrid system.

\subsection{Relay to destination VLC channel model}

In T2 time slot, the relay employs zero-forcing detection to decode the information within the received signal $y_{s r}$. Decoded bit stream from the received signal is modulated and DC bias is added to ensure that the produced signal is non negative, before being transmitted to the receiver. The transmitted optical signal from the LED source can be written as

$$
y_{t}=n p_{\text {led }} l,
$$

where $p_{\text {led }}$ denotes the power of a single LED, $n$ is the number of LEDs in LED array and $l$ denotes the DC bias added electrical modulated signal. There is a line of sight $(\mathrm{LoS})$ path from each LED in the LED array to the receiver and a diffuse path due to reflections from the room's surfaces. However, in this work we consider only the LoS propagation without considering reflections from the surrounding surfaces of room to avoid complications in the channel model. According to the work [16], the LoS visible light (VL) channel gain is defined as

$h_{v l c}=\left\{\begin{array}{lr}\frac{(m+1) A_{r}}{2 \pi d^{2}} \cos ^{m}(\phi) T_{s}(\psi) g(\psi) \cos \psi, & 0 \leq \psi \leq \psi_{c} \\ 0, & \psi>\psi_{c}\end{array}\right.$

where $A_{r}$ represents the physical area of photo-diode in the receiver, $c$ is the half angle of receiver's field of view, $\phi$ is the irradiation angle and $\psi$ is the angle of incident. $m$ Denotes the Lambertian index which is related to the half-intensity radiation angle $\phi_{1 / 2}$, by $m=-1 \log _{2}\left(\cos \left(\phi_{1 / 2}\right)\right) . T_{s}(\psi)$ is the gain of the optical filter and $g(\psi)$ denotes the non-imaging concentrator gain. The symbol $d$ denotes the distance between the LED array and the receiver. Therefore, the received signal at the destination receiver can be defined as

$$
y_{r d}=\eta p_{r} h_{v l c} l+n_{r d}
$$

where $\eta$ is the optical to electrical conversion efficiency at the destination receiver; $p_{r}=n p_{\text {led }}$ is the power of LED array; $h_{v l c}$ denotes the VLC channel power gain; $l$ is denoted as the DC bias added electrical modulated signal in the relay; and $n_{r d}$ represents the AWGN coming from thermal and shot noise in the room. However, clipping distortion needs to be avoided by having the 
input electrical signal within the region of LED operations. This can be achieved by adjusting the DC bias. The dynamic range of operations of LED can be denoted by [10] $D \triangleq I_{\max }-I_{\text {min }}$, where $I_{\max }$ represents the maximum input and $I_{\min }$ represents the minimum input of bias current. Thus $l$ can be given by,

$$
l=\left\{\begin{array}{l}
B-I_{\min }, \text { if } B<\frac{I_{\min }+I_{\max }}{2}, \\
I_{\max }-B, \text { if } B \geq \frac{I_{\min }+I_{\max }}{2} .
\end{array}\right.
$$

According to the work of [17], shot noise is the dominant noise source in the VLC. Thus, received SNR at the destination receiver can be expressed as

$$
\gamma_{r d}=\left(\eta p_{r} h_{v l c} l\right)^{2}+N_{0}^{\prime},
$$

where $\boldsymbol{N}_{\mathbf{0}}^{\prime}$ is the power of noise suffered by the destination receiver, which is located inside the room.

\section{Outage probability computation}

In this section we present the outage analysis of the proposed RFVLC hybrid communication model. The target transmission rate is expressed as $\mathrm{R}$, where $\mathrm{k}$ is the data rate per block per second. The outage probability of the base station to relay link can be approximately expressed as

$$
P_{\text {out }(S R)}=1-\exp \left(-\frac{N_{0}\left(2^{k R}-1\right)}{p_{s}\left|h_{s r}\right|^{2}}\right) .
$$

Outage probability of the source-to-relay link with the decoder effect of the decode-forward relay protocol can be written as

$$
P_{\text {out }(S R+D F)}=1-\left[\left(1-P_{\text {out }(S R)}\right)\left(1-S E R_{\text {th }}\right)\right],
$$

where

$$
S E R_{t h}=1-\sqrt{\frac{\gamma_{s r}}{1+\gamma_{s r}}} .
$$

Outage probability of relay to destination (R-D) VLC link can be written as

$$
P_{\text {out }(V L C)}=1-\exp \left(-\frac{N_{0}^{\prime}\left(2^{k R}-1\right)}{\left(\eta p_{r} h_{v l c} l\right)^{2}}\right) .
$$

Thus, the outage probability of the entire proposed RF-VLC hybrid scheme (source-relay-destination) can be given as

$$
P_{\text {out }(\text { hybrid })}=1-\left(\left(1-P_{\text {out }(V L C)}\right)\left(1-P_{\text {out }(S R+D F)}\right)\right) \text {. }
$$

Comparing with the general RF cooperative scheme, proposed RF-VLC hybrid cooperative scheme can potentially improve the network performance, especially for the case of limited RF bandwidth in the system. The performance of the proposed scheme is validated by the simulation results in Section 4 .

\section{Numerical results}

In this section, we present numerical results obtained to validate the above theoretical analysis. Unless otherwise explicitly specified, the main parameters of the VLC communication are set as $p_{\text {led }}=20 \mathrm{~mW} / \mathrm{A}, n=10, \psi=20 \mathrm{deg} ., \phi=20 \mathrm{deg} ., d=$ $1.5 m, A_{r}=0.04 m^{2}$ and $\eta=0.4 A / W$. Moreover, we assumed that the noise power of the VLC channel is equal to $10^{-3}$ in order to increase the noise in the VLC channel.

Figure 2, plots the SER for QPSK modulation in RFVLC hybrid system. As observed in Figure 2, proposed RFVLC hybrid scheme shows better SER performance compared to conventional RF co-

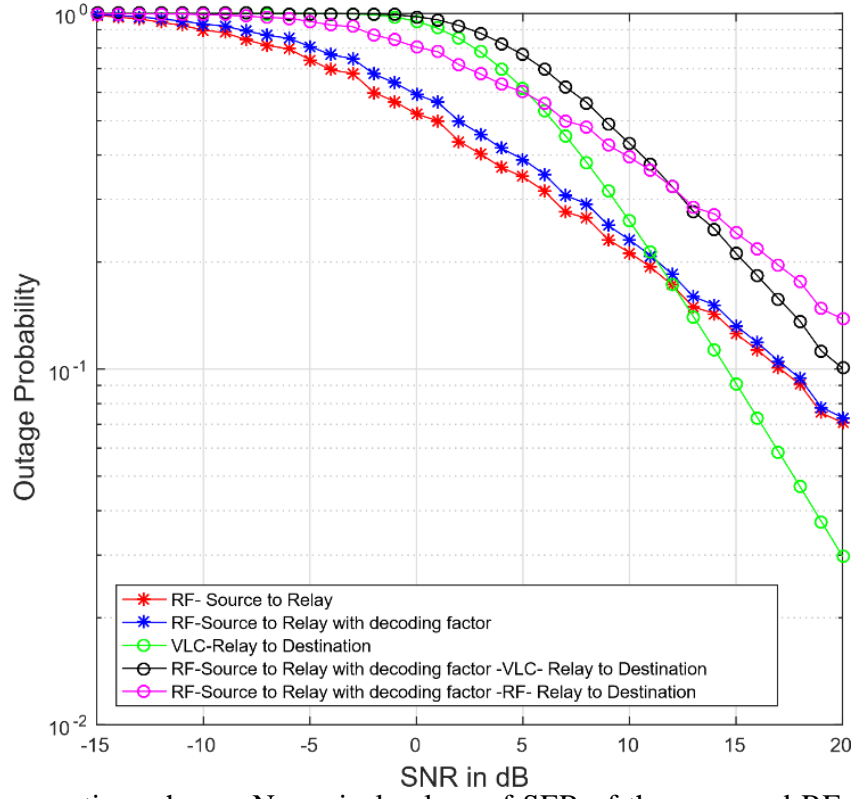

operative scheme. Numerical values of SER of the proposed RFVLC

Fig. 2: Comparison of SER of VLC relay to destination link, RF source to relay link, RF-VLC hybrid link and fully RF source to relay to destination link over Rayleigh flat fading with AWGN channels.

hybrid system and conventional RF cooperative system are included in Table I. According to the data represented in the table, SER gain of RF-VLC hybrid system over conventional RF cooperative system increases with the SNR value. SER value of the VLC relay-to-destination link is observed to be 0 when the SNR value falls below -9 . Though the two curves, RF source to relay link and RF-VLC source-to-relay-to-destination link in Figure 2 appears to be similar, there is a slight difference between the two curves. However, according to the results obtained, the proposed hybrid scheme shows approximately equal SER values as the RF source to relay link due to the high efficiency of VLC. Thus, SER performance of hybrid RF-VLC S-R-D system can be concluded to be better than the SER performance of conventional RF S-R-D cooperative system.

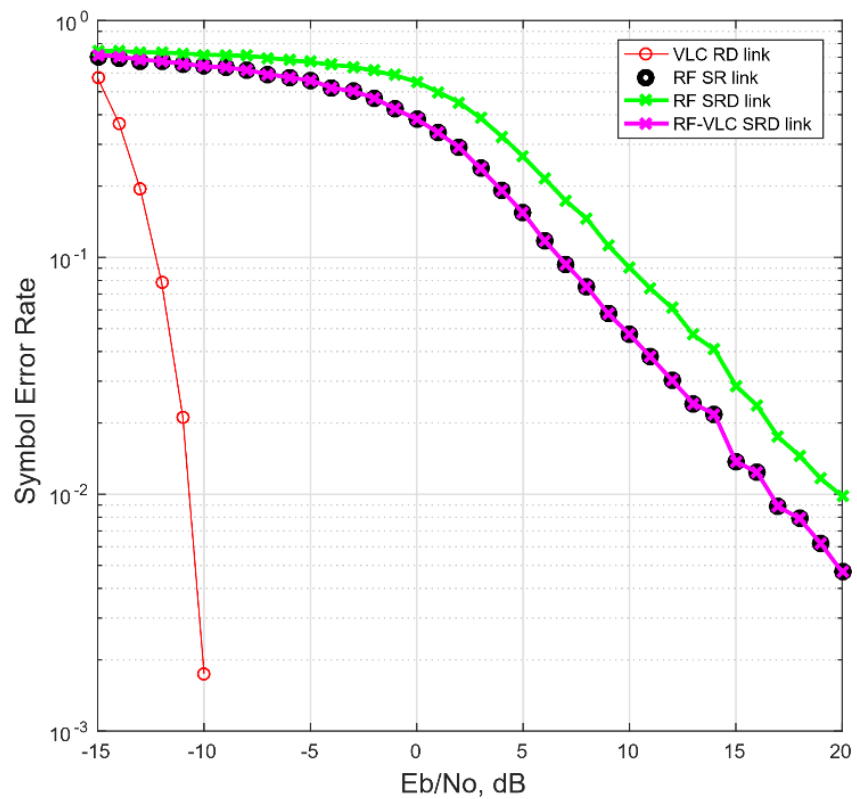

Fig. 3: Comparison of outage probability for proposed RF-VLC hybrid model with different RF schemes. 
Table I: Comparison of Symbol Error Rate in proposed RF-VLC hybrid system and conventional RF cooperative system

\begin{tabular}{|l|c|c|c|c|c|c|c|}
\hline SNR(dB) & -15 & -14 & -13 & -12 & -11 & -10 & -9 \\
\hline SER (RF-SR link) & 0.6943 & 0.6888 & 0.6687 & 0.6633 & 0.6557 & 0.6416 & 0.6300 \\
\hline SER (VLC RD link) & 0.5794 & 0.3896 & 0.2147 & 0.0925 & 0.0222 & 0.0034 & 0 \\
\hline SER (RF SR-RD link) & 0.7441 & 0.7429 & 0.7339 & 0.7258 & 0.7246 & 0.7152 & 0.7171 \\
\hline SER (RF-VLC Hybrid (S-R-D link)) & 0.7127 & 0.7004 & 0.6783 & 0.6675 & 0.6559 & 0.6416 & 0.6301 \\
\hline $\begin{array}{l}\text { SER gain of RF-VLC hybrid model over } \\
\text { RF S-R-D link }\end{array}$ & 0.0314 & 0.0425 & 0.0556 & 0.0583 & 0.0687 & 0.0736 & 0.087 \\
\hline
\end{tabular}

In Figure 3, the outage probability is plotted against the signal to noise ratio (SNR) of hybrid RF-VLC scheme with different communication schemes. The plots for five scenarios of data transmission have been analysed. The five scenarios considered are;

1) outage in source to relay RF link

2) outage in source to relay RF link with decoding factor

3) outage in relay destination VLC link

4) outage in combined RF-VLC hybrid scheme with decoding factor

5) outage in combined RF scheme with decoding factor

It is observed from Figure 3 that the VLC-RD link has better outage performance than the RF-SR link in higher SNR values. Moreover, Figure 3 clearly indicates that the outage performance of hybrid RF-VLC scheme is outperforming when compared with the RF cooperative scheme.

Therefore, it is observed that the hybrid RF-VLC system has better outage performance than RF communication system at higher SNR values. On considering the observations made from Figure 2 and Figure 3, hybrid RF-VLC system performance is better than RF cooperative communication system for both SER and Outage Probability, the performance measuring parameters considered in this paper. Therefore, as envisioned the seamless high speed data communication is enhanced by this model of hybrid RF-VLC system. Finally, the results obtained from Figure 2 and Figure 3 proves that the proposed RF-VLC hybrid communication system is superior to the full RF cooperative system.

\section{Conclusion}

In this paper we have proposed a downlink hybrid RF/VLC communication system to ensure high speed data to be received at a destination with the minimum use of RF spectrum. A case study of wireless communication in smart room has been considered in creating the system model. For simplicity we do not consider a direct link between the source and the destination in the system model. A RF link has been used in between the source and the relay, while a VLC link has been used in between the relay and the destination. The performance of proposed hybrid RF/VLC system is investigated based on the simulation results obtained for the parameters; SER and outage probability. Simulation results depicted that the SER of proposed hybrid RF-VLC system has a significant improvement in comparison with the conventional RFcooperative system. Moreover, the outage probability is found to be better in the proposed system as compared with the RF nonhybrid cooperative system. Thus, the overall observation from these numerical results obtained conforms the expected enhancement of the hybrid RF-VLC system's performance. Considering all the parameters, it is found that the hybrid RF-VLC system outperforms the conventional lone RF communication in smart room situation.

\section{Acknowledgment}

This work was funded, in part, by the framework of Competitiveness Enhancement Program of the National Research Tomsk Polytechnic University No. TPU-CEP-IC1012017. Authors also acknowledged the contribution of the COST Action on Inclusive Radio Communications (IRACON) No. CA15104.

\section{References}

[1] D. A. Basnayaka and H. Haas, "Design and analysis of a hybrid radio frequency and visible light communication system," IEEE Transactions on Communications, vol. 65, no. 10, pp. 4334-4347, 2017.

[2] S. Chedup, B. Subba, S. Dorji, T. D. P. Perera, A. Rajaram, and D. N. K. Jayakody, "Visible light energy harvesting in modern communication systems," IEEE International Conference on Electrical, Electronics, Computers, Communication, Mechanical and Computing (EECCMC). IEEE, 2017.

[3] D. N. K. Jayakody, J. Thompson, S. Chatzinotas, and S. Durrani, "Wireless Information and Power Transfer: A New Paradigm for Green Communications," Springer-Verlag New York, USA, April 2018.

[4] D. A. Basnayaka and H. Haas, "Hybrid RF and VLC systems: Improving user data rate performance of VLC systems," Vehicular Technology Conference (VTC Spring), 2015 IEEE 81st. IEEE, pp. 1-5, 2015.

[5] M. Kafafy, Y. Fahmy, M. Abdallah, and M. Khairy, "Power efficient downlink resource allocation for hybrid RF/VLC wireless networks," Wireless Communications and Networking Conference (WCNC), IEEE, 2017, pp. 1-6, 2017.

[6] M. B. Rahaim, A. M. Vegni, and T. D. Little, "A hybrid radio frequency and broadcast visible light communication system," GLOBECOM Workshops (GC Wkshps), IEEE, pp. 792-796, 2011.

[7] A. Jovicic, J. Li, and T. Richardson, "Visible light communication: opportunities, challenges and the path to market," IEEE Communications Magazine, vol. 51, no. 12, pp. 26-32, 2013.

[8] H. S. Khallaf, A. S. Ghazy, H. M. Shalaby, and S. S. Obayya, "Performance analysis of visible light communication systems over fading channels," Transparent Optical Networks (ICTON), $201719^{\text {th }}$ International Conference on. IEEE, pp. 1-4, 2017.

[9] G. Pan, J. Ye, and Z. Ding, "Secure hybrid VLC-RF systems with light energy harvesting," IEEE Transactions on Communications, vol. 65, no. 10, pp. 4348-4359, 2017.

[10] T. Rakia, H.-C. Yang, F. Gebali, and M.-S. Alouini, "Optimal design of dual-hop VLC/RF communication system with energy harvesting," IEEE Communications Letters, vol. 20, no. 10, pp. 1979-1982, 2016.

[11] T. Rakia, H.-C. Yang, F. Gebali, and M.-S. Alouini, "Dual-hop VLCIRF transmission system with energy harvesting relay under delay constraint," Globecom Workshops (GC Wkshps), IEEE, pp. 1-6, 2016.

[12] M. Hammouda, S. Akın, A. M. Vegni, H. Haas, and J. Peissig, "Link selection in hybrid RF/VLC systems under statistical queueing costraints," IEEE Transactions on Wireless Communications, vol.17, no. 04, pp.2738-2754, 2018.

[13] Y. Wang, X. Wu, and H. Haas, "Load balancing game with shadowing effect for indoor hybrid Li-Fi/RF networks, ”IEEE Transactions on Wireless Communications, vol. 16, no. 4, pp. 2366-2378, 2017.

[14] C. Chen, D. A. Basnayaka, and H. Haas, "Downlink performance of optical attocell networks," Journal of Lightwave Technology, vol. 34, no. 1, pp. 137-156, 2016.

[15] H. Kazemi, M. Safari, and H. Haas, "A wireless backhaul solution using visible light communication for indoor $\mathrm{Li}-\mathrm{Fi}$ attocell networks," International Conference on Communications (ICC), IEEE, pp. 1-7, 2017. 
[16] J. M. Kahn and J. R. Barry, "Wireless infrared communications," Proceedings of the IEEE, vol. 85, no. 2, pp. 265-298, 1997.

[17] T. Komine and M. Nakagawa, "Integrated system of white led visible light communication and power-line communication," IEEE Transactions on Consumer Electronics, vol. 49, no. 1, pp. 71-79, 2003. 